\title{
MEASUREMENT OF THE BACKGROUND ENERGY CONTENT OF Nd-GLASS PICOSECOND PULSES WITH SATURABLE ABSORBERS
}

\author{
J. WIEDMANN and A. PENZKOFER \\ Fachbereich Physik der Universität Regensburg, Germany
}

Received 16 January 1978

Revised manuscript received 13 February 1978

\begin{abstract}
The background energy content of a mode-locked Nd-glass laser is determined with photodetectors and saturable absorbers. By comparing the signal height of a fast detector with the readout of an integrating energy meter the noise energy $E_{\mathrm{u}_{2}}$ outside the rise-time of the fast detector is measured. The background energy $E_{\mathrm{u}_{1}}$ within the rise-time is analysed by transmission measurements through two subsequent absorber cells. The obtained mean background to pulse energy (and intensity) ratios of $\left(E_{\mathrm{u}_{1}} / E_{\mathrm{p}}\right)_{\mathrm{m}}=0.015 \pm 0.012\left(\left(I_{\mathrm{u}_{1}} / I_{0} \mathrm{~L}\right)_{\mathrm{m}}=1.8 \times 10^{-4} \pm 1.5 \times 10^{-4}\right)$ and $\left(E_{\mathrm{u}_{2}} / E_{\mathrm{p}}\right)_{\mathrm{m}}=0.05 \pm 0.01$ $\left(\left(I_{\mathrm{u}_{2}} / I_{0 \mathrm{~L}}\right)_{\mathrm{m}}=3 \times 10^{-5} \pm 6 \times 10^{-6}\right)$ indicate a high degree of mode-locking.
\end{abstract}

In mode-locked lasers the radiation is concentrated in a train of picosecond light pulses. The efficiency of mode-locking is characterized by the energy content in the train of picosecond pulses compared to the background energy (noise energy) between the pulses. Various techniques were applied to determine the signal to noise ratio of picosecond pulse trains. By overexposing fast photodetectors the background signal outside the pulse width of an oscilloscope-detector system could be displayed [1,2]. The contrast ratio of two-photon fluorescence traces was used as an indication of the mode-locking quality $[2,3]$. The efficiency of third harmonic generation $[4,5]$, of threephoton fluorescence $[2,6]$ and of four-photon parametric interaction [7] allowed the calculation of the background energy content. The transmission of picosecond pulse trains through saturable absorbers of various thickness [8] was applied to estimate the peak pulse intensity and the beam noise level. A direct measurement of the pulse duration and of the background energy was carried out with a picosecond streak camera [9]. The limited dynamic range reduced the background intensity resolution in the region following the intense picosecond pulse.

In this letter we describe a new technique to monitor the background energy content between two successive picosecond pulses. The background energy con- tent $E_{\mathrm{u} 2}$ outside the risetime $t_{\mathrm{r}}$ of a fast photodetector-oscilloscope system is measured by comparing the signal heights of a fast detector with that of an integrating detector. The detectors are calibrated by removing the background energy with a saturable absorber of low small signal transmission $T_{0}$. The background energy $E_{\mathrm{u} 1}$ within the integration time $t_{\mathrm{r}}$ of the fast photodetector is analyzed by transmission measurements through two bleachable dye cells in series. In the first cell the energy transmission is reduced by background absorption, while the input into the second cell is nearly free of background and a higher energy transmission results. A comparison of the energy transmission allows to determine the background content.

The situation is illustrated in fig. 1. The separated part of the pulse train with duration $t_{\mathrm{s}}=10 \mathrm{~ns}$ (equal to the cavity round trip time) is shown in a semilogarithmic plot in fig. 1a. As an example it is assumed that the selected part consists of a single pulse of peak intensity $I_{0 \mathrm{~L}}$ and of duration $\Delta t_{\mathrm{L}}=6 \mathrm{ps}$ (value not resolved in the picture) and a background trace of intensity $I_{\mathrm{u}}=6 \times 10^{-4} I_{0 \mathrm{~L}}$. The integrated laser energy $E_{\mathrm{L}}(t)=\int_{0}^{\infty} 2 \pi r \int_{0}^{\mathrm{t}} I_{\mathrm{L}}\left(r, t^{\prime}\right) \mathrm{d} t^{\prime} \mathrm{d} r$ of the single pulse and the background is shown in fig. 1b. For the assumed numbers of fig. 1a the background energy $E_{\mathrm{u}}$ is equal to the pulse energy $E_{\mathrm{p}}$. The total energy content 


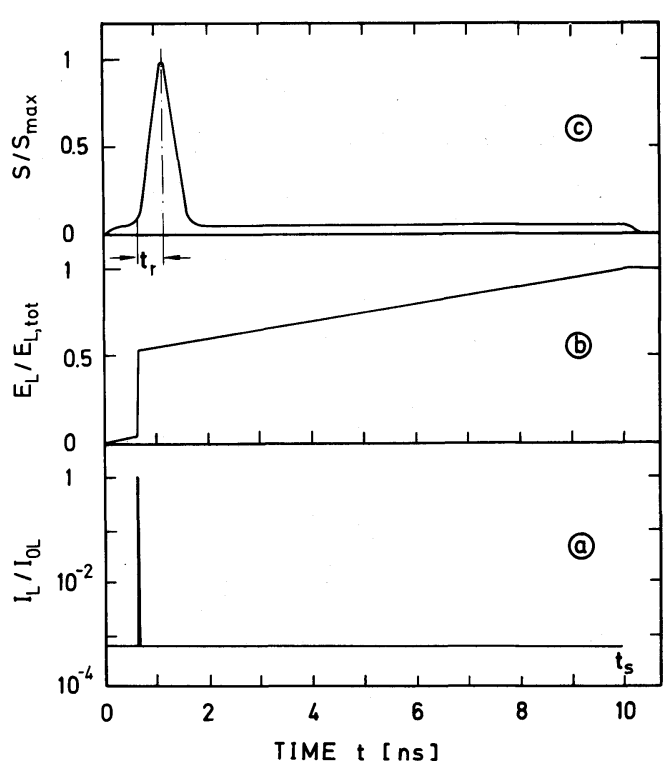

Fig. 1. Illustration of pulse and background detection. (a) Semilogarithmic plot of normalized laser intensity $I_{\mathrm{L}} / I_{0 \mathrm{~L}}$ versus time $t$. Duration of picosecond pulse $\Delta t_{\mathrm{L}}=6 \mathrm{ps}$ (not resolved); background level $I_{\mathrm{u}}=6 \times 10^{-4} I_{0 \mathrm{~L}}$. (b) Normalized energy content $E_{\mathrm{L}} / E_{\mathrm{L}}$, tot of laser light depicted in (a) $\left(E_{\mathrm{p}}=E_{\mathrm{u}}\right)$. (c) Oscilloscope trace of the output of a fast detector corresponding to the light signal of (a).

$E_{\mathrm{L}, \text { tot }}=E_{\mathrm{p}}+E_{\mathrm{u}}$ may be measured with an integrating photodetector, a detector of slow risetime, or an energy meter. In fig. $1 c$ the corresponding oscilloscope trace for a fast photodetector (integration time $t_{\mathrm{r}} \approx$ $500 \mathrm{ps}$ ) is depicted. The peak signal height $S_{\max }$ is proportional to the pulse energy $E_{\mathrm{p}}$ and the background energy $E_{\mathrm{u} 1}$ within the time $t_{\mathrm{r}}$. The background level $S_{\mathrm{u} 2}$ is a factor of twenty lower than the peak value and is hard to distinguish from the baseline in real experiments, i.e. only background energies $E_{\mathrm{u}} \geqslant$ $E_{\mathrm{p}}$ can be resolved.

The background energy content $E_{\mathrm{u} 2}$ outside the risetime of the fast detector (period $t_{\mathrm{s}}-t_{\mathrm{r}}$ ) is given by

$E_{\mathrm{u} 2}=E_{\mathrm{L}, \text { tot }}-E_{\text {fast }}=\left(\frac{E_{\mathrm{L}, \text { tot }}}{E_{\text {fast }}}\right) E_{\text {fast }}$.

$E_{\text {fast }}$ is proportional to the signal height $S_{\text {max }}$ of the fast detector and comprises the pulse energy $E_{\mathrm{p}}$ and the noise energy $E_{\mathrm{u} 1}$,

$E_{\text {fast }}=E_{\mathrm{p}}+E_{\mathrm{u} 1}$.

The background energy content $E_{\mathrm{u} 1}$ is determined by measuring the energy transmission through two bleachable dye cells with fast photodetectors. The energy transmission $T_{\mathrm{E}}^{(1)}=S_{\max , \text { out }}^{(1)} / S_{\max , \text { in }}^{(1)}$ through the first cell is determined by the energy transmission $T_{\mathrm{E}, \mathrm{P}}^{(1)}$ of the picosecond light pulse and $T_{\mathrm{u} 1}^{(1)}$ of the background within the rise-time $t_{\mathrm{r}}$ :

$T_{\mathrm{E}}^{(1)}=\frac{E_{\mathrm{p}} T_{\mathrm{E}, \mathrm{P}}^{(1)}+E_{\mathrm{u} 1} T_{\mathrm{u} 1}^{(1)}}{E_{\mathrm{P}}+E_{\mathrm{u} 1}}$.

Solving eq. (3) to $E_{\mathrm{u} 1}$ leads to

$E_{\mathrm{u} 1}=E_{\mathrm{P}} \frac{T_{\mathrm{E}, \mathrm{P}}^{(1)}-T_{\mathrm{E}}^{(1)}}{T_{\mathrm{E}}^{(1)}-T_{\mathrm{u} 1}^{(1)}}$,

$T_{\mathrm{u} 1}^{(1)}$ is approximately equal to $T_{0}$.

The background energy content $E_{\mathrm{u} 2}$ outside the rise-time of the fast detector (region $t_{\mathrm{s}}-t_{\mathrm{r}}$ ) is found by inserting eqs. (2) and (4) into eq. (1).

$E_{\mathrm{u} 2}=E_{\mathrm{P}}\left(\frac{E_{\mathrm{Ltot}}}{E_{\text {fast }}}-1\right) \frac{T_{\mathrm{E}, \mathrm{P}}^{(1)}-T_{\mathrm{u} 1}^{(1)}}{T_{\mathrm{E}}^{(1)}-T_{\mathrm{u} 1}^{(1)}}$.

For small background energies $T_{\mathrm{E}, \mathrm{P}}^{(1)}$ is only slightly larger than $T_{\mathrm{E}}^{(1)}$ and $E_{\mathrm{u} 2} / E_{\mathrm{P}}$ is approximately equal to $E_{\text {u2 }} / E_{\text {fast }}$.

Assuming homogeneous temporal distribution of the background light, the intensity ratios are found to be

$\frac{I_{\mathrm{u} 1}}{I_{0 \mathrm{~L}}}=\frac{E_{\mathrm{u} 1}}{E_{\mathrm{P}}} \frac{\Delta t_{\mathrm{L}}}{t_{\mathrm{r}}}$

and

$\frac{I_{\mathrm{u} 2}}{I_{0 \mathrm{~L}}}=\frac{E_{\mathrm{u} 2}}{E_{\mathrm{P}}} \frac{\Delta t_{\mathrm{L}}}{t_{\mathrm{s}}-t_{\mathrm{r}}}$.

The energy transmission $T_{\mathrm{E}, \mathrm{P}}^{(1)}$ of the single picosecond light pulse (duration $\Delta t_{\mathrm{L}}$ ) without background is calculated from transmission measurements of the light beam through the subsequent second dye cell. The background energy is reduced in the first cell and is negligibly small in the second cell for proper selected $T_{0}$-values of the absorbers. The energy transmission $T_{E}^{(2)}$ through the second cell is determined by the picosecond light pulse that passed through the first cell $\left(T_{\mathrm{E}}^{(2)} \approx T_{\mathrm{E}, \mathrm{P}}^{(2)}\right)$. By comparing with theory the energy transmission $T_{E}^{(1)}$ is obtained from the measured energy transmission $T_{E}^{(2)}$.

In fig. 2 the energy transmission $T_{\mathrm{E}}$ of picosecond 


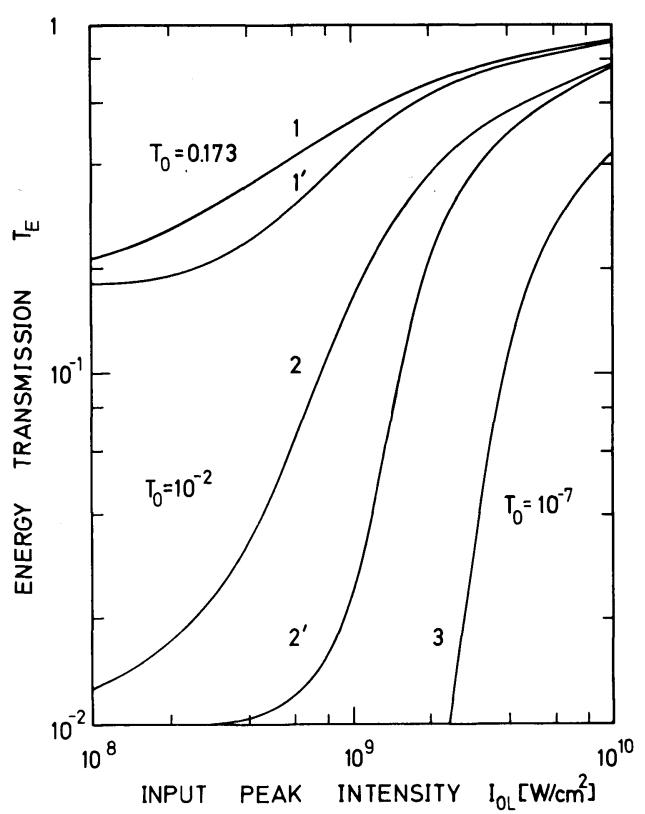

Fig. 2. Energy transmission of picosecond light pulses through saturable absorbers. 1 and 2, energy transmission of gaussian input pulses ( $\left.\Delta t_{\mathrm{L}}=6 \mathrm{ps}\right)$ through a first dye cell. $1^{\prime}$ and $2^{\prime}$, energy transmission of the output pulses (additionally attenuated to $I=0.76 I_{\text {out }}$ ) through a subsequent second dye cell. $I_{0 \mathrm{~L}}$ is the input peak intensity to the first dye cell. 3 , transmission of gaussian shaped pulses through an absorber with $T_{0}=10^{-7}$. The dye parameters are recovery time $\tau=9 \mathrm{ps}$ and absorption cross-section $\sigma=1.84 \times 10^{-16} \mathrm{~cm}^{2}$.

pulses through saturable absorbers is plotted. The curves are calculated using a two-level model for the transient absorption of the dyes as described in refs. [10-12]. The dye parameters used in the calculations were ground state recovery time $\tau=9$ ps and absorption cross-section $\sigma=1.84 \times 10^{-16} \mathrm{~cm}^{2}$ (Kodak dye solution No. 9860) [13]. Curve 3 represents the energy transmission of picosecond pulses $\left(\Delta t_{\mathrm{L}}=6 \mathrm{ps}\right.$, gaussian shape) through an absorber cell with a low intensity transmission of $T_{0}=10^{-7}$. A dye solution with this $T_{0}$-value was applied to remove the background signal for the calibration of the detectors. Curves 1 and 2 show the energy transmission $T_{\mathrm{E}, \mathrm{P}}^{(1)}$ of gaussian shaped (temporal and spatial) pulses of 6 ps duration through an absorber (first cell in our experiments) with $T_{0}=0.173$ and 0.01 , respectively. The transmitted pulses are reduced in intensity, shortened in time, and reshaped.

Part of the energy of these pulses is coupled out for the transmission measurement. Only 76 per cent of the energy of the output pulses enters into a subsequent dye cell of equal small signal transmission. The energy transmission $T_{\mathrm{E}, \mathrm{p}}^{(2)}$ of these pulses versus the input peak intensity to the first cell is shown by the curves $1^{\prime}$ and $2^{\prime}$. The $T_{\mathrm{E}, P}^{(2)}$ values (curves $1^{\prime}$ and $2^{\prime}$ ) are practically equal to the experimentally measured $T_{\mathrm{E}}^{(2)}$. values. Due to the background reduction in the first dye cell, we estimate with the aid of eq. (3) and fig. 2 $T_{\mathrm{E}}^{(2)} \geqslant 0.98 T_{\mathrm{E}, \mathrm{P}}^{(2)}$ for $E_{\mathrm{u} 1} / E_{\mathrm{p}} \leqslant 0.15$ at $T_{0}=0.173$ and for $E_{\mathrm{u} 1} / E_{\mathrm{p}} \leqslant 2$ at $T_{0}=0.01$ when $I_{0 \mathrm{~L}} \geqslant 3 \times 10^{9} \mathrm{~W} /$ $\mathrm{cm}^{2}$. (In our experiments we found $E_{\mathrm{u} 1} / E_{\mathrm{p}} \leqslant 0.15$, see later.)

In fig. 3 , the energy transmission of the picosecond light pulses through the first absorber $T_{\mathrm{E}, \mathrm{P}}^{(1)}$ is plotted versus the energy transmission $T_{\mathrm{E}, \mathrm{P}}^{(2)}$ through the subsequent second absorber ( $T_{0}=0.173$ in both cells). The curves $3\left(\Delta t_{\mathrm{L}}=2 \mathrm{ps}\right)$ and $4\left(\Delta t_{\mathrm{L}}=16 \mathrm{ps}\right)$ show that the ratio $T_{\mathrm{E}, \mathrm{P}}^{(1)} / T_{\mathrm{E}}^{(2)} \mathrm{p}$ is only slightly dependent on the pulse duration in the region between 2 ps and 16 ps. The absolute transmission at a fixed intensity depends more strongly on the pulse duration [10-12]. The curves 3 and 4 are calculated for incident gaussian

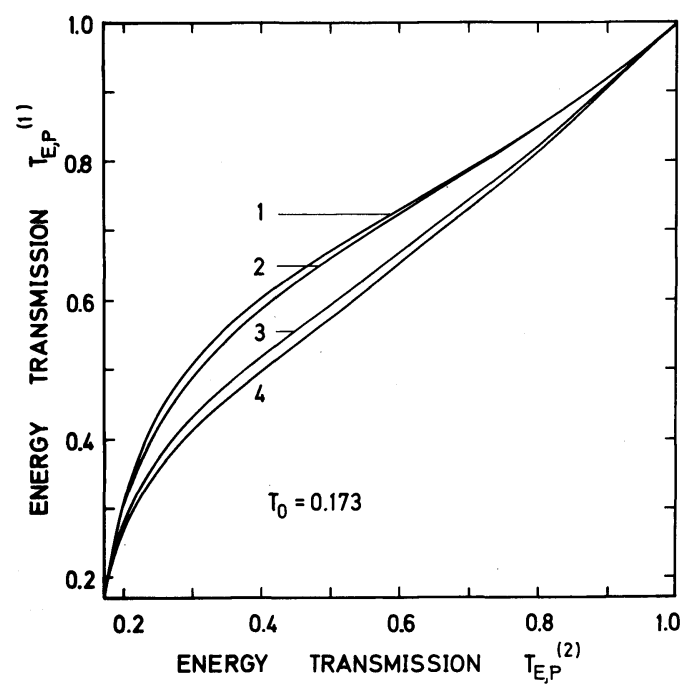

Fig. 3. Energy transmission of picosecond pulses through a first dye cell versus transmission through a subsequent absorber of equal $T_{0}$. $\left(I_{\text {in }}\right.$ (second cell) $=0.76 I_{\text {out }}$ (first cell)). Incident pulse parameters are: 1 , temporal gaussian and spatial rectangular shape $\left(\Delta t_{\mathrm{L}}=6 \mathrm{ps}\right) ; 2$, temporal gaussian and spatial truncated gaussian shape (truncated at $I_{\mathrm{L}}(r)=0.5 I_{0 \mathrm{~L}}$; $\Delta t_{\mathrm{L}}=6 \mathrm{ps}$ ); 3 and 4 , temporal and spatial gaussian shape with $\Delta t_{\mathrm{L}}=2$ ps (curve 3 ) and $\Delta t_{\mathrm{L}}=16$ ps (curve 4 ). 
shaped pulses (temporal and spatial). Curve 2 depicts the dependence of $T_{\mathrm{E}, \mathrm{P}}^{(1)}$ on $T_{\mathrm{E}, \mathrm{P}}^{(2)}$ for an input pulse $\left(\Delta t_{\mathrm{L}}=6 \mathrm{ps}\right)$ of temporal gaussian and spatial truncated gaussian shape (truncation at half the peak intensity value). It is only slightly different from the case of a spatial rectangular and temporal gaussian pulse (see curve 1).

In our experiments we used a mode-locked Ndphosphate glass laser. The mode-locking dye cell was contacted to the 100 per cent mirror of the oscillator to avoid satellite pulses. A single pulse was selected from the pulse train with an electrooptical shutter (opening time $=$ cavity round trip time $t_{\mathrm{s}}=10 \mathrm{~ns}$ ). The selected pulse and the background trace were increased in energy by a laser amplifier. The single pulses had a duration of approximately 6 ps (FWHM) and a spectral width of $\Delta \widetilde{\nu} \approx 3 \AA$ (nearly bandwidthlimited). The energy of the pulses behind the amplifier was approximately $2 \mathrm{~mJ}$.

The experimental arrangement for the background detection is depicted in fig. 4 . The dye sample $\mathrm{S} 1$ with $T_{0}=10^{-7}$ (Kodak dye solution No. 9860) is only in the path of the laser beam for the relative energy calibration of the fast photodetector PD1 to the integrating energy meter EM (removal of background). The detectors PD1 (fast) and EM (slow) measure $E_{\text {fast }}$ and $E_{\mathrm{L}, \text { tot }}$ and allow to determine $E_{\mathrm{u} 2} / E_{\text {fast }}=$ $\left(E_{\mathrm{L}, \text { tot }}-E_{\text {fast }}\right) / E_{\text {fast }}$. The background energy $E_{\mathrm{u} 1}$ is obtained by measuring the energy transmission through the dye cells $\mathrm{S} 2\left(T_{\mathrm{E}}^{(1)} ; T_{0}=0.173\right)$ and $\mathrm{S} 3\left(T_{\mathrm{E}}^{(2)} ; T_{0}=\right.$ 0.173 ) with the photodetectors PD1, PD2 and PD3. Spatailly truncated light beams were used by inserting

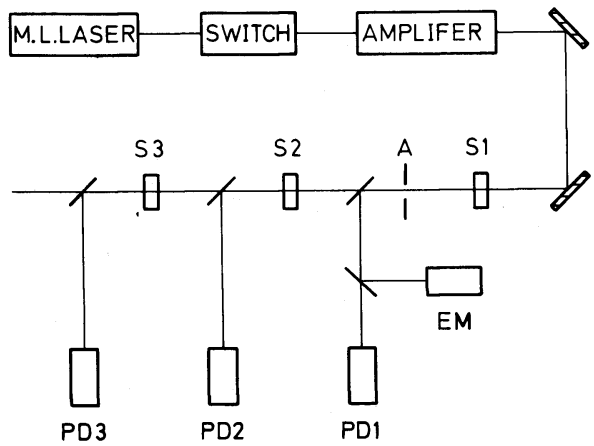

Fig. 4. Experimental set-up. $\mathrm{S}_{1}-\mathrm{S}_{3}$ saturable absorbers; PD1-PD3, fast photo-detectors; EM, integrating energy meter; A, aperture. an aperture $\mathrm{A}$ into the light path. (Uncertainties due to deviations from spatial gaussian beam profiles are thereby excluded.) Curve 2 of fig. 3 was applied to determine $T_{\mathrm{E}, \mathrm{P}}^{(1)}$ from $T_{\mathrm{E}, \mathrm{P}}^{(2)}\left(T_{\mathrm{E}}^{(2)}\right.$ is set equal to $T_{\mathrm{E}, \mathrm{P}}^{(2)}$. Light pulses with input peak intensities $I_{0 \mathrm{~L}} \geqslant 3 \times 10^{9}$ $\mathrm{W} / \mathrm{cm}^{2}$ were used to increase the accuracy of the background detection (large $T_{\mathrm{E}}^{(1)}$-values in eqs. (4) and (5)).

With the described technique, the background energy content of a laser pulse is obtained in a single shot. In our measurements we found $E_{\mathrm{u} 1} / E_{\mathrm{p}}$ (eq. (4)) in the range between -0.15 and +0.15 and $E_{\mathrm{u} 2} / E_{\mathrm{p}}$ (eq. (5)) in the region between -0.05 and +0.15 for 95 per cent of all measured shots. The number of evaluated shots was approximately $n \approx 50$. The mean values and the standard deviations of the single shots [14] are $E_{\mathrm{u} 1} / E_{\mathrm{p}}=0.015 \pm 0.08$ and $E_{\mathrm{u} 2} / E_{\mathrm{p}}=0.05 \pm$ 0.06 . Assuming a homogeneous distribution of noise the corresponding values for the intensity ratios are $I_{\mathrm{u} 1} / I_{0 \mathrm{~L}}=1.8 \times 10^{-4} \pm 1 \times 10^{-3}$ and $I_{\mathrm{u} 2} / I_{0 \mathrm{~L}}=3 \times$ $10^{-5} \pm 4 \times 10^{-5}$. The numbers are presented in the form $x=\bar{x} \pm \sigma_{S}$, where $\bar{x}=(1 / n) \Sigma_{i=1}^{n} x_{i}$ is the mean value and $\sigma_{\mathrm{S}}=\left[\sum_{i=1}^{n}\left(x_{i}-\bar{x}\right)^{2} /(n-1)\right]^{1 / 2}$ is the standard deviation of the single shot.

The scatter of the single shot data is caused besides by fluctuations of the noise - by the unaccuracy of the signal heights of the photodetectors (see the calculated negative values of $E_{\mathrm{u} 1} / E_{\mathrm{p}}$ and $\left.E_{\mathrm{u} 2} / E_{\mathrm{p}}\right)$. These accidental erros of the single shot technique are reduced by averaging over many shots. The standard error of the mean $\sigma_{\mathrm{m}}$ is a factor of $\sqrt{ } n$ smaller than the standard deviation of the single shot: $\sigma_{\mathrm{m}}=\sigma_{\mathrm{S}} /$ $n^{1 / 2}$. In our experiments $(n \approx 50)$ the mean values and their standard errors $\left(x_{\mathrm{m}}=\bar{x} \pm \sigma_{\mathrm{m}}\right)$ are $\left(E_{\mathrm{u} 1} /\right.$ $\left.E_{\mathrm{p}}\right)_{\mathrm{m}}=0.015 \pm 0.012\left(\left(I_{\mathrm{u} 1} / I_{\mathrm{p}}\right)_{\mathrm{m}}=1.8 \times 10^{-4} \pm\right.$ $\left.1.5 \times 10^{-4}\right)$ and $\left(E_{\mathrm{u} 2} / E_{\mathrm{p}}\right)_{\mathrm{m}}=0.05 \pm 0.01\left(\left(I_{\mathrm{u} 2} / I_{\mathrm{p}}\right)_{\mathrm{m}}\right.$ $\left.=3 \times 10^{-5} \pm 6 \times 10^{-6}\right)$. The noise levels $I_{\mathrm{u} 1}$ and $I_{\mathrm{u} 2}$ seem to be equal within the standard error of the mean. In cases where two pulses were present within $t_{\mathrm{s}}$, the background energy content remained in the same range. This fact indicates that even in modelocking situations where two spikes survive the noise reduction is very effective.

In conclusion we wish to state that the new technique of background detection is accurate and easy to perform. The background energy is determined in a single laser shot. The background energy content may be measured by separating a small part of the laser pulse and focusing the selected part of the beam to 
the necessary intensity; the main part of the pulse remains for the experiment under study. The obtained mean values of $\left(I_{\mathrm{u} 1} / I_{0 \mathrm{~L}}\right)_{\mathrm{m}}=1.8 \times 10^{-4} \pm 1.5 \times$ $10^{-4}$ and $\left(I_{\mathrm{u} 2} / I_{0 \mathrm{~L}}\right)_{\mathrm{m}}=3 \times 10^{-5}+6 \times 10^{-6}$ indicate a good quality of mode-locking of the investigated $\mathrm{Nd}$-glass laser system.

The authors are indepted to Professor M. Maier for valuable discussions.

\section{References}

[1] G. Dube, Appl. Phys. Letters 18 (1971) 69.

[2] D. von der Linde, IEEE J. Quantum Electron. QE-8 (1972) 328.

[3] M.A. Duguay, J.W. Hansen and S.L. Shapiro, IEEE J. Quantum Electron. QE-6 (1970) 725.
[4] C.C. Wang and E.L. Baardsen, Appl. Phys. Letters 15 (1969) 396.

[5] R.C. Eckardt and C.H. Lee, Appl. Phys. Letters 15 (1969) 425.

[6] P.M. Rentzepis, C.J. Mitschele and A.C. Saxman, Appl. Phys. Letters 17 (1970) 122.

[7] D.H. Auston, Appl. Phys. Letters 18 (1971) 249.

[8] R.J. Harrach, T.D. Mac Vicar, G.I. Kachen and L.L. Steinmetz, Opt. Commun. 5 (1972) 175.

[9] D.J. Bradley, B. Liddy, A.G. Roddie, W. Sibbett and W.E. Sleat, Opt. Commun. 3 (1971) 426.

[10] A. Penzkoper, D. von der Linde and A. Laubereau. Opt. Commun. 4 (1972) 377.

[11] A. Penzkofer, D. von der Linde, A. Laubereau and W. Kaiser, Appl. Phys. Letters 20 (1972) 351.

[12] A. Penzkoper, Opto-Electronics 6 (1974) 87.

[13] Eastman Kodak dye No. A 9860, data release.

[14] J. Topping, Errors of observation and their treatment (Chapman and Hall Ltd., London, 1969). 